Tohoku Math. J

58 (2006), 101-121

\title{
TORIC FANO THREE-FOLDS WITH TERMINAL SINGULARITIES
}

\author{
ALEXANDER M. KASPRZYK \\ (Received February 13, 2004, revised August 18, 2004)
}

\begin{abstract}
This paper classifies all toric Fano 3-folds with terminal singularities. This is achieved by solving the equivalent combinatorial problem; that of finding, up to the action of $G L(3, Z)$, all convex polytopes in $\boldsymbol{Z}^{3}$ which contain the origin as the only non-vertex lattice point. We obtain, up to isomorphism, 233 toric Fano 3 -folds possessing at worst $\boldsymbol{Q}$-factorial singularities (of which 18 are known to be smooth) and 401 toric Fano 3-folds with terminal singularities that are not $Q$-factorial.
\end{abstract}

0. Background and Introduction. A toric variety of dimension $n$ over an algebraically closed field $k$ is a normal variety $X$ that contains a torus $T \cong\left(k^{*}\right)^{n}$ as a dense open subset, together with an action $T \times X \rightarrow X$ of $T$ on $X$ that extends the natural action of $T$ on itself.

Let $M \cong Z^{n}$ be the lattice of characters of the torus $T$, with dual lattice $N=\operatorname{Hom}(M, Z)$. Then every toric variety $X$ has an associated fan $\Delta$ in $N \otimes Q$. The converse also holds; to any fan $\Delta$ there is an associated toric variety $X(\Delta)$. For details on the construction and deeper properties of this crucial bijection see [Dan78, Oda78, Ful93, Ewa96, Cox02].

A normal variety $X$ is a Fano variety if some multiple of the anticanonical divisor $-K_{X}$ is an ample Cartier divisor. Thus $X$ is $\boldsymbol{Q}$-Gorenstein. There is a well-known description of what it means for a toric variety $X$ to be a Fano variety in terms of its fan $\Delta$ : Let $\left\{\rho_{i}\right\}_{i \in I}$ be the set of rays of $\Delta$. For each $i \in I$ there exists a unique primitive lattice element of $\rho_{i}$, which by a traditional abuse of notation we continue to denote $\rho_{i}$. Then $X$ is Fano if and only if $\left\{\rho_{i}\right\}_{i \in I}$ correspond to the vertices of a convex polytope in $N$ (see [Dan78, Ewa96]).

Fano varieties are important for several reasons. In particular they play a significant role in the Minimal Model Program (see [Wiś02, FS03]). Many general results concerning smooth toric Fano $n$-folds are known [Wiś02]; of particular relevance, it has been shown that there exist precisely 18 smooth toric Fano 3-folds.

A normal variety $X$ is $Q$-factorial if every prime divisor $\Gamma \subset X$ has a positive integer multiple $c \Gamma$ which is a Cartier divisor. Once again, in the toric case there exists a well-known description in terms of the fan. The toric variety $X$ is $\boldsymbol{Q}$-factorial if and only if the fan $\Delta$ is simplicial (see [Oda78, Dai02]).

We say that a fan $\Delta$ is terminal if each cone $\sigma \in \Delta$ satisfies the following:

(i) the lattice points $\rho_{1}, \ldots, \rho_{k}$ corresponding to the rays of $\sigma$ are contained in an affine hyperplane $H:=\left\{n \in N_{\boldsymbol{Q}} \mid m(n)=1\right\}$ for some $m \in M_{\boldsymbol{Q}}$

2000 Mathematics Subject Classification. Primary 14J45; Secondary 14J30, 14M25, 52B20.

Key words and phrases. Toric, Fano, 3-folds, terminal singularities, convex polytopes. 
(ii) with the exception of 0 , there are no other elements of the lattice $N$ in the part of $\sigma$ under or on $H$; we have,

$$
N \cap \sigma \cap\left\{n \in N_{Q} \mid m(n) \leq 1\right\}=\left\{0, \rho_{1}, \ldots, \rho_{k}\right\} .
$$

A toric variety $X$ is terminal (i.e., has terminal singularities [Rei83]) if and only if the fan $\Delta$ is terminal.

Terminal singularities play an important role in birational geometry. A great deal of classification results exist in various cases; for example the results of [Mor82, Rei83, MS84, MMM88]. In [Mor85] it was shown that, with two exceptions, isolated canonical cyclic quotient singularities in dimension three are all either Gorenstein or terminal. In [Rei85] Reid addresses the issue of classifying 3-fold terminal singularities. In the notation of [Rei85, Theorem $6.1, \mathrm{II}]$ we are in the case $(1 / r)(a,-a, 1,0 ; 0)$.

We are now in a position to state our aim, a complete classification of all toric Fano 3 -folds with terminal singularities, in terms of a purely combinatorial problem. Namely, we wish to find, up to the action of $G L(3, \boldsymbol{Z})$, all convex lattice polytopes in $\boldsymbol{Z}^{3}$ which contain only the origin as a non-vertex lattice point (by which we mean that no lattice points lie on the surface of the polytope other than the vertices, and no lattice points are contained in the interior of the polytope other than the origin).

An equivalent restatement for 2-folds can be found in [Ewa96, pp. 192-193]; precisely five polytopes are found, of which two are minimal (the Fano triangle and the Fano square, which make an appearance in Section 3) and one is maximal, in the sense of Definitions 3.2 and 4.1. The approach used for this classification relies on the basic result that, up to the action of $G L(2, \boldsymbol{Z})$, there is a unique lattice point free triangle (namely conv $\left\{0, e_{1}, e_{2}\right\}$ ). This fails to hold in dimension three (see [Sca85]). It is also worth observing that in dimension two all polytopes are simplicial (and hence the corresponding toric variety is, at worst, $\boldsymbol{Q}$-factorial), something which is clearly not the case in dimension three.

The classification presented in this paper is inspired by the work of A. Borisov and L. Borisov [BB, BB93]. Results given in [BB93, Bor00] assure us that a finite classification is possible. The combinatorial approach we adopt is based on that formulated in [BB]. In this unpublished work, the essential steps described can be outlined thus:

(i) Observe that every polytope can be "grown" from a "minimal" polytope.

(ii) These minimal polytopes divide into tetrahedra and non-tetrahedra.

(iii) The minimal tetrahedra can be classified in terms of their barycentric coordinates.

(iv) The minimal non-tetrahedra can be determined directly.

(v) A recursive algorithm can be written, allowing a computer to "grow" these minimal polytopes and hence classify all polytopes of interest.

The result of Proposition 1.4 is a specific case of [BB93, Proposition 3]. However the proof presented here is of an elementary combinatorial nature, in keeping with the style of the remainder of this paper. In addition the results of Table 4 are obtained more explicitly than in [BB93]; again the justification for repeating these results lies in the methods used to obtain them. With a nice restatement of Proposition 1.4 (concerning tetrahedra containing one 
non-vertex lattice point) we obtain a result which closely mirrors one of [Sca85] (concerning tetrahedra containing no non-vertex lattice points), although once more the methods of proof are very different.

For practical reasons the final classification is not contained in this paper, but has been made available on the internet (see the end of Section 4 for the address). We conclude this introduction by presenting a summary of the main features of this list (see also Table 7).

THEOREM 0.1. Up to isomorphism, there exist exactly 233 toric Fano 3-folds having at worst $Q$-factorial singularities, of which 18 are smooth. There exist additional 401 having terminal singularities that are not $\boldsymbol{Q}$-factorial.

There exist 12 minimal cases with at worst $\boldsymbol{Q}$-factorial singularities: eight with Picard number 1, two with Picard number 2, and two with Picard number 3. There exists one minimal case with terminal singularities, corresponding to a polytope with 5 vertices.

There exist nine maximal cases, corresponding to polytopes with 8 (three occurrences), 9, 10 (two occurrences), 11, 12 and 14 vertices. Only those with 8 vertices are $Q$-factorial.

The author would like to express his gratitude to Professor Alexander Borisov for making [BB] available; the current paper relies heavily upon many of the ideas developed in this unpublished work. Special thanks to Dr. Gregory Sankaran for introducing me to this problem, and for his invaluable explanations and advice.

The author wishes to acknowledge funding by an Engineering and Physical Sciences Research Council (EPSRC) Mathematics CASE studentship from GCHQ.

1. Classifying the tetrahedra: The barycentric coordinates. Throughout this paper we take $N:=Z_{\geq 0}=\{0,1,2, \ldots\}$. When we refer to $e_{1}, e_{2}$ or $e_{3}$ we mean the standard basis elements of $Z^{3}$. If $x$ is a point in $Z^{3}$, by $x_{1}, x_{2}$ and $x_{3}$ we mean the integers such that $x=\left(x_{1}, x_{2}, x_{3}\right)$. For any $q \in \boldsymbol{Q}$ we define $\lfloor q\rfloor:=\max \{a \in \boldsymbol{Z} \mid a \leq q\}$ and $\lceil q\rceil:=\min \{a \in \boldsymbol{Z} \mid a \geq q\}$. The fractional part of $q$, which we shall denote $\{q\}$, is given by $q-\lfloor q\rfloor$.

We will make frequent appeals to the following well-known result:

LEMMA 1.1. Any lattice point free triangle with vertices $\left\{0, x_{1}, x_{2}\right\} \subset Z^{3}$ is equivalent under the action of $G L(3, Z)$ to the triangle with vertices $\left\{0, e_{1}, e_{2}\right\}$.

Let $\left\{x_{1}, \ldots, x_{4}\right\} \subset \boldsymbol{Z}^{3}$ be the lattice point vertices of a tetrahedron containing the origin. Let $\mu_{1}, \ldots, \mu_{4} \in \boldsymbol{Q}$ give the (unique) barycentric coordinate of the origin with respect to the $x_{i}$; i.e.,

$$
\begin{aligned}
& \mu_{1} x_{1}+\cdots+\mu_{4} x_{4}=0 \\
& \mu_{1}+\cdots+\mu_{4}=1 \\
& \mu_{1} \geq 0, \ldots, \mu_{4} \geq 0
\end{aligned}
$$

Choose $\lambda_{1}, \ldots, \lambda_{4} \in N$ coprime such that $\mu_{i}=\lambda_{i} / h$, where $h=\lambda_{1}+\cdots+\lambda_{4}$.

LEMMA 1.2. For any $\kappa \in\{2, \ldots, h-2\}$ we have that $\sum_{i=1}^{4}\left\{\lambda_{i} \kappa / h\right\} \in\{1,2,3\}$. 
Proof. Since $\sum_{i=1}^{4} \lambda_{i} \kappa / h=\kappa \in N$ it follows that $\sum_{i=1}^{4}\left\{\lambda_{i} \kappa / h\right\} \in\{0,1,2,3\}$. Suppose for some $\kappa \in\{2, \ldots, h-2\},\left\{\lambda_{i} \kappa / h\right\}=0$ for $i=1,2,3,4$. We have that $h \mid \kappa \lambda_{i}$ for each $i$, so let $p$ be a prime such that $p \mid h$, so that $h=p^{r} h^{\prime}$ where $p \nmid h^{\prime}$. Then $p^{r} \mid \kappa \lambda_{i}$. Suppose that $p^{r} \nmid \kappa$. Then $p \mid \lambda_{i}$ for each $i$. Hence $p \mid \operatorname{gcd}\left\{\lambda_{1}, \ldots, \lambda_{4}\right\}=1$, a contradiction. Thus $p^{r} \mid \kappa$. By induction on the prime divisors of $h$ we see that $h \mid \kappa$, so in particular $h \leq \kappa$, which is a contradiction.

For convenience we make the following definition:

DEFINITION 1.3. We say a tetrahedron is Fano if the vertices lie at lattice points and the only non-vertex lattice point it contains is the origin, which lies strictly in the interior of the tetrahedron.

PROPOSITION 1.4. If the tetrahedron associated with the $\lambda_{i}$ is Fano then

(i) $\sum_{i=1}^{4}\left\{\lambda_{i} \kappa / h\right\}=2$ for all $\kappa \in\{2, \ldots, h-2\}$, and

(ii) $\operatorname{gcd}\left\{\lambda_{i}, \lambda_{j}\right\}=1$ for $i \neq j$.

Proof. Let the $\lambda_{i}$ be associated with a Fano tetrahedron. Since the origin is strictly in the interior the $\lambda_{i}$ are all non-zero. By Lemma 1.2 we only need to consider the cases where $\sum_{i=1}^{4}\left\{\lambda_{i} \kappa / h\right\}=1$ or 3 . But if $\sum_{i=1}^{4}\left\{\lambda_{i} \kappa / h\right\}=3$ for some $\kappa$, then $\sum_{i=1}^{4}\left\{\lambda_{i}(h-\kappa) / h\right\}=1$.

Suppose for some $\kappa \in\{2, \ldots, h-2\}$ the sum is 1 . Let $\chi_{i}=\left\{\lambda_{i} \kappa / h\right\}$. Then $\left(\chi_{1}, \ldots, \chi_{4}\right)$ is the (unique) barycentric coordinate for some point in the tetrahedron. We shall show that it is a non-vertex lattice point not equal to the origin.

We have that $\sum_{i=1}^{4}\left\lfloor\lambda_{i} \kappa / h\right\rfloor x_{i}$ is a lattice point, call it $a \in Z^{3}$. We also have that $\sum_{i=1}^{4}\left(\lambda_{i} \kappa / h\right) x_{i}=0$. Thus

$$
\sum_{i=1}^{4} \chi_{i} x_{i}=\sum_{i=1}^{4} \frac{\lambda_{i} \kappa}{h} x_{i}-\sum_{i=1}^{4}\left\lfloor\frac{\lambda_{i} \kappa}{h}\right\rfloor x_{i}=-a \in Z^{3} .
$$

By the uniqueness of barycentric coordinates we have that $-a$ is a non-vertex point, since each $\chi_{i}<1$. Furthermore suppose $-a=0$, so that $\chi_{i}=\lambda_{i} / h$ for $i=1,2,3,4$. For each $i, \lambda_{i} \kappa / h-\left\lfloor\lambda_{i} \kappa / h\right\rfloor=\lambda_{i} / h$, so we obtain that $\left\lfloor\lambda_{i} \kappa / h\right\rfloor=\lambda_{i}(\kappa-1) / h$ and hence that $h \mid \lambda_{i}(\kappa-1)$. As in the proof of Lemma 1.2 we find that $h \mid \kappa-1$, and so in particular $h+1 \leq \kappa$. This contradicts our range for $\kappa$. Hence $-a$ must be a non-vertex, non-zero lattice point in the tetrahedron, contradicting our hypothesis.

Now suppose for a contradiction that $\operatorname{gcd}\left\{\lambda_{1}, \lambda_{2}\right\} \neq 1$. We have

$$
\frac{\lambda_{3}}{\operatorname{gcd}\left\{\lambda_{1}, \lambda_{2}\right\}} x_{3}+\frac{\lambda_{4}}{\operatorname{gcd}\left\{\lambda_{1}, \lambda_{2}\right\}} x_{4}=-\frac{\lambda_{1}}{\operatorname{gcd}\left\{\lambda_{1}, \lambda_{2}\right\}} x_{1}-\frac{\lambda_{2}}{\operatorname{gcd}\left\{\lambda_{1}, \lambda_{2}\right\}} x_{2} \in \boldsymbol{Z}^{3} .
$$

Since the triangle with vertices $\left\{0, x_{3}, x_{4}\right\}$ is lattice point free, by Lemma 1.1 there exists an element of $G L(3, \boldsymbol{Z})$ mapping $x_{3} \mapsto e_{1}$ and $x_{4} \mapsto e_{2}$. Hence it must be that $\operatorname{gcd}\left\{\lambda_{1}, \lambda_{2}\right\} \mid \lambda_{3}$ and $\operatorname{gcd}\left\{\lambda_{1}, \lambda_{2}\right\} \mid \lambda_{4}$, thus $\operatorname{gcd}\left\{\lambda_{1}, \ldots, \lambda_{4}\right\} \neq 1$.

COROLlaRY 1.5. Let $\left(\lambda_{1}, \ldots, \lambda_{4}\right)$ be associated with a Fano tetrahedron. Then

(i) $\sum_{i=1}^{4}\left\lceil\lambda_{i} \kappa / h\right\rceil=\kappa+2$ for all $\kappa \in\{2, \ldots, h-2\}$, and 
(ii) $\operatorname{gcd}\left\{\lambda_{i}, h\right\}=1$ for $i=1, \ldots, 4$.

Proof. Proposition 1.4 tells us that $\sum_{i=1}^{4}\left\{\lambda_{i} \kappa / h\right\}=2$ for all $\kappa \in\{2, \ldots, h-2\}$. Since $\left\{\lambda_{i} \kappa / h\right\}<1$ it must be that $\left\{\lambda_{i} \kappa / h\right\}=0$ for at most one value of $i$. We shall show that in fact $\left\{\lambda_{i} \kappa / h\right\} \neq 0$ for all $i=1, \ldots, 4$.

Suppose (with possible relabelling of the indices) that:

$$
\left\{\frac{\lambda_{4} \kappa}{h}\right\}=0 \text {. }
$$

Then $\sum_{i=1}^{3}\left\{\lambda_{i} \kappa / h\right\}=2$ and hence $\sum_{i=1}^{3}\left\{\lambda_{i}(h-\kappa) / h\right\}=1$. We obtain

$$
\sum_{i=1}^{4}\left\{\frac{\lambda_{i}(h-\kappa)}{h}\right\}=1 .
$$

But equation (1.2) contradicts Proposition 1.4. Hence,

$$
\left\{\frac{\lambda_{i} \kappa}{h}\right\} \neq 0 \quad \text { for } i=1, \ldots, 4 .
$$

By hypothesis we have $\left\{\lambda_{1} \kappa / h\right\}+\cdots+\left\{\lambda_{4} \kappa / h\right\}=2$, and by definition $\lambda_{1} \kappa / h+\cdots+$ $\lambda_{4} \kappa / h=\kappa$. Hence we obtain $\left\lfloor\lambda_{1} \kappa / h\right\rfloor+\cdots+\left\lfloor\lambda_{4} \kappa / h\right\rfloor=\kappa-2$. This, combined with (1.3), tells us that $\left\lfloor\lambda_{i} \kappa / h\right\rfloor=\left\lceil\lambda_{i} \kappa / h\right\rceil-1$ for $i=1, \ldots, 4$. This proves the first part of the claim. Finally suppose that, for some $i, \operatorname{gcd}\left\{\lambda_{i}, h\right\} \neq 1$. Then taking $\kappa=h / \operatorname{gcd}\left\{\lambda_{i}, h\right\} \in$ $\{2, \ldots, h-2\}$ we have $\left\{\lambda_{i} \kappa / h\right\}=0$. Hence $\operatorname{gcd}\left\{\lambda_{i}, h\right\}=1$.

Although not required, it is worth observing the similarity between Corollary 1.5 and the following:

PROPOSITION 1.6 ([Sca85]). Let a lattice point tetrahedron containing no non-vertex lattice points have the vertices of Lemma 2.3 with $x, y, z \geq 1$. Let $d:=x+y+z-1$. Then

(i) $\lceil\kappa x / d\rceil+\lceil\kappa y / d\rceil+\lceil\kappa z / d\rceil=\kappa+2$ for all $\kappa \in\{1, \ldots, d-1\}$, and

(ii) $\operatorname{gcd}\{x, d\}=\operatorname{gcd}\{y, d\}=\operatorname{gcd}\{z, d\}=1$.

Let $h \geq 4$. By making use of Corollary 1.5 we can construct bounds on the $\lambda_{i}$. We may assume without loss of generality that $\lambda_{1} \leq \cdots \leq \lambda_{4}$. For each $\kappa \in\{2, \ldots, h-2\}$ and each $i$ let $a_{i}^{(\kappa)}=\left\lceil\lambda_{i} \kappa / h\right\rceil$. The following conditions are immediate:

$$
\begin{aligned}
& a_{1}^{(\kappa)} \leq \cdots \leq a_{4}^{(\kappa)}, \\
& a_{1}^{(\kappa)}+\cdots+a_{4}^{(\kappa)}=\kappa+2, \\
& \left(a_{1}^{(2)}, a_{2}^{(2)}, a_{3}^{(2)}, a_{4}^{(2)}\right)=(1,1,1,1) .
\end{aligned}
$$

We have also that $(h / \kappa)\left(a_{i}^{(\kappa)}-1\right)<\lambda_{i}<(h / \kappa) a_{i}^{(\kappa)}$, and so

$$
h \max _{2 \leq n \leq \kappa} \frac{1}{n}\left(a_{i}^{(n)}-1\right)<\lambda_{i}<h \min _{2 \leq n \leq \kappa} \frac{1}{n} a_{i}^{(n)} .
$$


Recalling that $\lambda_{i} / h=\mu_{i}$ gives us

$$
\begin{aligned}
\frac{1}{\kappa}\left(a_{i}^{(\kappa)}-1\right) & <\mu_{i}<\frac{1}{\kappa} a_{i}^{(\kappa)}, \\
\max _{2 \leq n \leq \kappa} \frac{1}{n}\left(a_{i}^{(n)}-1\right) & <\mu_{i}<\min _{2 \leq n \leq \kappa} \frac{1}{n} a_{i}^{(n)} .
\end{aligned}
$$

This suggests a recursive method of determining an upper bound for $h$. Assume $h \geq 4$ is associated with a Fano tetrahedron. Then it is possible to construct a sequence $\left\{\left(a_{1}^{(\kappa)}, \ldots, a_{4}^{(\kappa)}\right)\right\}_{2 \leq \kappa \leq h-2}$ satisfying the conditions (1.4) and (1.6) for all $\kappa \in\{2, \ldots, h-2\}$. Moreover we have that for each $\kappa \in\{2, \ldots, h-3\}$ there exists some $i \in\{1, \ldots, 4\}$ such that

$$
a_{j}^{(\kappa+1)}= \begin{cases}a_{j}^{(\kappa)} & \text { for } j \neq i, \\ a_{j}^{(\kappa)}+1 & \text { for } j=i .\end{cases}
$$

LEMMA 1.7. Let $a, k \in N$ be such that $a<k$. Then $a / k>a /(k+1)$ and $a / k<$ $(a+1) /(k+1)$.

An immediate consequence of Lemma 1.7 is that

$$
\frac{1}{\kappa+1} a_{i}^{(\kappa+1)}=\frac{1}{\kappa+1}\left(a_{i}^{(\kappa)}+1\right) \geq \min _{2 \leq n \leq \kappa} \frac{1}{n} a_{i}^{(n)}
$$

and hence, using (1.5) and (1.6), we obtain

$$
\frac{1}{\kappa+1}\left(a_{i}^{(\kappa+1)}-1\right)=\frac{1}{\kappa+1} a_{i}^{(\kappa)}<\mu_{i}<\min _{2 \leq n \leq \kappa+1} \frac{1}{n} a_{i}^{(n)}=\min _{2 \leq n \leq \kappa} \frac{1}{n} a_{i}^{(n)} .
$$

Thus we have the requirement that

$$
\frac{1}{\kappa+1} a_{i}^{(\kappa)}<\min _{2 \leq n \leq \kappa} \frac{1}{n} a_{i}^{(n)} .
$$

Conditions (1.4) and (1.7) are independent of $h$, so by writing a simple recursive function on a computer it is possible to test these conditions for large values of $\kappa$, using all the sequences obtained for $\kappa$ to check whether a sequence exists for $\kappa+1$. If no such sequence exists we have found an upper bound for $h$, namely $h \leq \kappa+2$.

It is worth observing that this method for finding a bound for $h$ really does do that; when all possible sequences have terminated it is impossible to proceed any further. No a priori guarantee that this search along all possible sequences will terminate has been given here.

It is also worth noting that the bound this method gives is not the tightest, but this deficiency is balanced by the fact that it providing a technique which is independent of $h$.

This yields a bound for $h \leq 30$. Proposition 1.8 now follows from Proposition 1.4 by the easy task of checking all possible $\lambda_{i}$ up to this bound. An alternative proof of Proposition 1.8 can be found in [BB93].

Proposition 1.8. Let $\lambda_{1} \leq \cdots \leq \lambda_{4}$ be associated with a Fano tetrahedron. Then $\left(\lambda_{1}, \ldots, \lambda_{4}\right)$ is equal to one of the following:
$(1,1,1,1)$
$(1,1,1,2)$
$(1,3,4,5)$
$(2,3,5,7)$
$(1,1,2,3)$
$(1,2,3,5)$
$(3,4,5,7)$. 
2. Classifying the tetrahedra: The coordinates of the vertices. Let $\left\{x_{1}, \ldots, x_{4}\right\} \subset$ $Z^{3}$ be the lattice point vertices of a Fano tetrahedron. Assume that the indices have been chosen such that $\lambda_{1} \leq \cdots \leq \lambda_{4}$. We represent this tetrahedron by the $3 \times 4$ matrix $\left(x_{1}^{t} \ldots x_{4}^{t}\right)$, where $x_{i}^{t}$ denotes the vertex $x_{i}$ regarded as a column vector.

Proposition 2.1. Let $\lambda_{1} \leq \cdots \leq \lambda_{4}$ be associated with a Fano tetrahedron. Then, by means of the action of $G L(3, Z)$, we can transform the tetrahedron to the form

$$
\left(\begin{array}{cccc}
1 & 0 & k^{\prime \prime} \lambda_{4}-a \lambda_{1} & -k^{\prime \prime} \lambda_{3}-b \lambda_{1} \\
0 & 1 & k^{\prime} \lambda_{4}-a \lambda_{2} & -k^{\prime} \lambda_{3}-b \lambda_{2} \\
0 & 0 & k \lambda_{4} & -k \lambda_{3}
\end{array}\right)
$$

where $a, b \in \boldsymbol{Z}, a>0$ are such that $a \lambda_{3}+b \lambda_{4}=1$, and $k, k^{\prime}, k^{\prime \prime} \in N$ are such that

$$
\begin{aligned}
0 & \leq k^{\prime \prime} \lambda_{4}-a \lambda_{1}<k \lambda_{4}, \\
\text { and } \quad 0 & \leq k^{\prime} \lambda_{4}-a \lambda_{2}<k \lambda_{4}
\end{aligned}
$$

with one of these inequalities equal to zero only if $\lambda_{4}=1$.

PROOF. By virtue of Lemma 1.1 we may assume without loss of generality that our tetrahedron has vertices $\left\{e_{1}, e_{2}, x, y\right\}$ with $\lambda_{1} e_{1}+\lambda_{2} e_{2}+\lambda_{3} x+\lambda_{4} y=0$. Thus we see that $\lambda_{3} x_{3}=-\lambda_{4} y_{3}$, and so $y_{3}=-\left(\lambda_{3} / \lambda_{4}\right) x_{3} \in Z$. Hence $\lambda_{4} \mid \lambda_{3} x_{3}$, but $\operatorname{gcd}\left\{\lambda_{3}, \lambda_{4}\right\}=1$ and so it must be that $\lambda_{4} \mid x_{3}$. Thus there exists some $k \in \boldsymbol{Z}$ such that

$$
x_{3}=k \lambda_{4}, \quad y_{3}=-k \lambda_{3} .
$$

We may take $x_{3}$ positive, and so $k \in N$.

We also have that $\lambda_{2}+\lambda_{3} x_{2}+\lambda_{4} y_{2}=0$, so that $\lambda_{3} x_{2}+\lambda_{4} y_{2}=-\lambda_{2}$. Now since $\operatorname{gcd}\left\{\lambda_{3}, \lambda_{4}\right\}=1$ there exist $a, b \in Z, a>0$ such that $\lambda_{3} a+\lambda_{4} b=1$. This gives us that $\lambda_{3}\left(-\lambda_{2} a\right)+\lambda_{4}\left(-\lambda_{2} b\right)=-\lambda_{2}$, so that $\lambda_{3}\left(x_{2}+a \lambda_{2}\right)+\lambda_{4}\left(y_{2}+b \lambda_{2}\right)=0$. Thus there exists some $k^{\prime} \in \boldsymbol{Z}$ such that

$$
x_{2}=k^{\prime} \lambda_{4}-a \lambda_{2}, \quad y_{2}=-k^{\prime} \lambda_{3}-b \lambda_{2} .
$$

Similarly we obtain that there exists some $k^{\prime \prime} \in \boldsymbol{Z}$ such that

$$
x_{1}=k^{\prime \prime} \lambda_{4}-a \lambda_{1}, \quad y_{1}=-k^{\prime \prime} \lambda_{3}-b \lambda_{1} .
$$

By applying

$$
\left(\begin{array}{lll}
1 & 0 & c \\
0 & 1 & d \\
0 & 0 & 1
\end{array}\right) \in G L(3, Z)
$$

for suitably chosen $c, d \in Z$, we can arrange matters so that (with possible relabelling of $k^{\prime}$ and $k^{\prime \prime}$ )

$$
\begin{aligned}
& 0 \leq k^{\prime} \lambda_{4}-a \lambda_{2}<k \lambda_{4}, \\
& 0 \leq k^{\prime \prime} \lambda_{4}-a \lambda_{1}<k \lambda_{4} .
\end{aligned}
$$


Now suppose that $k^{\prime} \lambda_{4}-a \lambda_{2}=0$. Since $\operatorname{gcd}\left\{\lambda_{2}, \lambda_{4}\right\}=1$, there must exist some constant $m \in \boldsymbol{Z}$ such that $k^{\prime}=m \lambda_{2}$ and $a=m \lambda_{4}$. In particular, this gives us that $\lambda_{4}\left(m \lambda_{3}+b\right)=1$, so that $\lambda_{4}=1$. Similarly, if $k^{\prime \prime} \lambda_{4}-a \lambda_{1}=0$.

The exceptional case in Proposition 2.1 occurring when $\lambda_{1}=\cdots=\lambda_{4}=1$ will be dealt with now.

PROPOSITION 2.2. Using the notation introduced above, the only exceptional case is given, up to equivalence, by the tetrahedron with vertices $\left\{e_{1}, e_{2}, e_{3},-e_{1}-e_{2}-e_{3}\right\}$.

PROOF. Using the notation introduced in the proof of Proposition 2.1, we may take $a=1, b=0$ and so taking $k^{\prime} \lambda_{4}-a \lambda_{2}=0$ implies that $k^{\prime}=1$. Thus we see that our tetrahedron has the form

$$
\left(\begin{array}{cccc}
1 & 0 & k^{\prime \prime}-1 & -k^{\prime \prime} \\
0 & 1 & 0 & -1 \\
0 & 0 & k & -k
\end{array}\right)
$$

where $k^{\prime \prime}$ and $k$ are to be determined.

The triangle defined by the origin, the first and the third vertices in the above matrix is lattice point free. Thus

$$
\operatorname{det}\left(\begin{array}{cc}
1 & k^{\prime \prime}-1 \\
0 & k
\end{array}\right)= \pm 1
$$

This forces $k=1$ and the resulting tetrahedron is equivalent to that given in the statement.

The following two results are taken from [Sca85]. A proof is given for the first result because we need to know explicitly the steps required for the transformation.

LEMMA 2.3 (cf. [Sca85]). A lattice point tetrahedron containing no non-vertex lattice points can, by means of a translation and the action of $G L(3, Z)$, be transformed to the form

$$
\left(\begin{array}{llll}
1 & 0 & 0 & x \\
0 & 1 & 0 & y \\
0 & 0 & 1 & z
\end{array}\right)
$$

where $x, y, z \in Z, x, y \geq 0, z \geq 1$.

PROOF. By applying a translation if necessary and considering Lemma 1.1, we may assume without loss of generality that the tetrahedron is in the form

$$
\left(\begin{array}{llll}
0 & 1 & 0 & x \\
0 & 0 & 1 & y \\
0 & 0 & 0 & z
\end{array}\right)
$$

where $z \geq 1$, but the conditions on $x$ and $y$ remain to be determined.

Let $x \mapsto x(\bmod z)$ and $y \mapsto y(\bmod z)$. Observe that this is equivalent to the (left) action of

$$
\left(\begin{array}{lll}
1 & 0 & a \\
0 & 1 & b \\
0 & 0 & 1
\end{array}\right) \in G L(3, Z)
$$


for suitably chosen $a, b \in Z$. Thus we can assume that $0 \leq x<z$ and $0 \leq y<z$. Suppose that $z<x+y$. Then set

$$
\mu_{1}:=1-\mu_{2}-\mu_{3}-\mu_{4}, \quad \mu_{2}:=1-\frac{x}{z}, \quad \mu_{3}:=1-\frac{y}{z}, \quad \mu_{4}:=\frac{1}{z} .
$$

Clearly $\sum \mu_{i}=1$, and $\mu_{2}, \mu_{3}, \mu_{4} \geq 0$. We have also that $\mu_{1}=(x+y-z-1) / z \geq 0$. But then

$$
\mu_{1}\left(\begin{array}{l}
0 \\
0 \\
0
\end{array}\right)+\mu_{2}\left(\begin{array}{l}
1 \\
0 \\
0
\end{array}\right)+\mu_{3}\left(\begin{array}{l}
0 \\
1 \\
0
\end{array}\right)+\mu_{4}\left(\begin{array}{l}
x \\
y \\
z
\end{array}\right)=\left(\begin{array}{l}
1 \\
1 \\
1
\end{array}\right)
$$

gives us a non-vertex lattice point in the interior of the tetrahedron, a contradiction. Thus it must be that $z \geq x+y$.

Finally we apply the unimodular transformation $z \mapsto-x-y+z+1$ which yields the result.

Proposition 2.4 (cf. [Sca85], Simplification of Howe's Theorem). Let a lattice point tetrahedron containing no non-vertex lattice points have the vertices of Lemma 2.3 with $x, y, z \geq 1$. Then $\{x, y, z\} \cap\{1\} \neq \emptyset$.

Let us now consider a Fano tetrahedron presented in the form given in Proposition 2.1. In addition we shall assume thst we are not looking at the case handled in Proposition 2.2. The tetrahedron with vertices given by $\left\{0, e_{1}, e_{2},(x, y, z)\right\}$, where

$$
x:=k^{\prime \prime} \lambda_{4}-a \lambda_{1} \geq 1, \quad y:=k^{\prime} \lambda_{4}-a \lambda_{2} \geq 1, \quad z:=k \lambda_{4} \geq 1,
$$

is lattice point free. By following the proof of Lemma 2.3 we see that it is equivalent to

$$
\left(\begin{array}{cccc}
1 & 0 & 0 & x \\
0 & 1 & 0 & y \\
0 & 0 & 1 & z-x-y+1
\end{array}\right)
$$

and that $z \geq x+y$. Proposition 2.4 tells us that $\{x, y, z-x-y+1\} \cap\{1\} \neq \emptyset$. Thus

$$
\begin{aligned}
& \text { either } \quad k^{\prime \prime}=\left(1+a \lambda_{1}\right) / \lambda_{4} \in Z \quad \text { if and only if } \quad x=1 \\
& \text { or } k^{\prime}=\left(1+a \lambda_{2}\right) / \lambda_{4} \in \boldsymbol{Z} \quad \text { if and only if } \quad y=1
\end{aligned}
$$

or $\quad k-k^{\prime}-k^{\prime \prime}=-a\left(\lambda_{1}+\lambda_{2}\right) / \lambda_{4} \in Z$ if and only if $\quad z-x-y+1=1$.

The result of applying this to the barycentric coordinates found in Proposition 1.8 is given in Table 1 . Observe that the only cases of ambiguity are for $(1,1,1,1)$ and $(1,1,1,2)$.

PROPOSITION 2.5. Let $\lambda_{1} \leq \cdots \leq \lambda_{4}$ be associated with a Fano tetrahedron presented in the form given in Proposition 2.1. Then

$$
\begin{aligned}
0 & \leq k \lambda_{3}-k^{\prime \prime} \lambda_{3}-b \lambda_{1}<k \lambda_{3} \\
\text { and } \quad & 0 \leq k \lambda_{3}-k^{\prime} \lambda_{3}-b \lambda_{2}<k \lambda_{3}
\end{aligned}
$$


TABLE 1. The values depending on $a$.

\begin{tabular}{|c|c|c|c|c|c|}
\hline$\left(\lambda_{1}, \lambda_{2}, \lambda_{3}, \lambda_{4}\right)$ & $a$ & $b$ & $\left(1+a \lambda_{1}\right) / \lambda_{4}$ & $\left(1+a \lambda_{2}\right) / \lambda_{4}$ & $a\left(\lambda_{1}+\lambda_{2}\right) / \lambda_{4}$ \\
\hline \hline$(1,1,1,1)$ & 1 & 0 & \multicolumn{2}{|c|}{2} & 2 \\
\hline$(1,1,1,2)$ & 1 & 0 & \multicolumn{2}{|c|}{1} & 1 \\
\hline$(1,1,2,3)$ & 2 & -1 & \multicolumn{2}{|c|}{1} & - \\
\hline$(1,2,3,5)$ & 2 & -1 & - & 1 & - \\
\hline$(1,3,4,5)$ & 4 & -3 & 1 & - & - \\
\hline$(2,3,5,7)$ & 3 & -2 & 1 & - & 3 \\
\hline$(3,4,5,7)$ & 3 & -2 & - & - & - \\
\hline
\end{tabular}

with one of these inequalities equal to zero only if $\lambda_{3}=1$, in which case the tetrahedron is equivalent either to that given in Proposition 2.2 or to

$$
\left(\begin{array}{llll}
1 & 0 & 1 & -1 \\
0 & 1 & 1 & -1 \\
0 & 0 & 2 & -1
\end{array}\right)
$$

ProOF. Since $a \lambda_{3}+b \lambda_{4}=1$ we have that $a=\left(1-b \lambda_{4}\right) / \lambda_{3}$. By substituting this into equation (2.1a) we obtain $\lambda_{1} / \lambda_{4} \leq k^{\prime \prime} \lambda_{3}+b \lambda_{1}<k \lambda_{3}+\lambda_{1} / \lambda_{4}$. Splitting this into two inequalities yields:

$$
\begin{aligned}
& k \lambda_{3}-k^{\prime \prime} \lambda_{3}-b \lambda_{1}>-\lambda_{1} / \lambda_{4} \\
& \text { and } \quad k \lambda_{3}-k^{\prime \prime} \lambda_{3}-b \lambda_{1} \leq k \lambda_{3}-\lambda_{1} / \lambda_{4} .
\end{aligned}
$$

Recall that $\lambda_{1} / \lambda_{4} \in(0,1]$. Hence we have that $0 \leq k \lambda_{3}-k^{\prime \prime} \lambda_{3}-b \lambda_{1}<k \lambda_{3}$. If instead we start with equation (2.1b) we derive that $0 \leq k \lambda_{3}-k^{\prime} \lambda_{3}-b \lambda_{2}<k \lambda_{3}$.

Now suppose that $k \lambda_{3}-k^{\prime \prime} \lambda_{3}-b \lambda_{1}=0$. Then we have that $\left(k-k^{\prime \prime}\right) \lambda_{3}=b \lambda_{1}$, and since $\operatorname{gcd}\left\{\lambda_{1}, \lambda_{3}\right\}=1$ there must exist some $c \in \boldsymbol{Z}$ such that $k-k^{\prime \prime}=c \lambda_{1}$ and $b=c \lambda_{3}$. But then $a \lambda_{3}+c \lambda_{3} \lambda_{4}=1$, which forces $\lambda_{3}=1$ (as required). The only cases where $\lambda_{3}=1$ are when $a=1, b=0$. Hence $k=k^{\prime \prime}$.

There are two possible choices for $\lambda_{4}$. First consider the case where $\lambda_{4}=1$. We have that $k \geq k^{\prime \prime}+k^{\prime}-2$, and $k^{\prime} \geq 2$. Thus $k^{\prime}=2$. Hence we see that our Fano tetrahedron is equivalent to the form

$$
\left(\begin{array}{cccc}
1 & 0 & -1 & 0 \\
0 & 1 & 1 & -2 \\
0 & 0 & k & -k
\end{array}\right)
$$

The triangle with vertices given by the origin, and the second and fourth column of the above matrix is lattice point free. By Lemma 1.1 it must be that $k=1$, which gives a tetrahedron equivalent to that derived in Proposition 2.2. 
Finally, consider the case where $\lambda_{4}=2$. We have that $k \geq k^{\prime \prime}+k^{\prime}-1$, and $k^{\prime} \geq 1$. Thus $k^{\prime}=1$. Hence we see that our Fano tetrahedron is equivalent to the form

$$
\left(\begin{array}{cccc}
1 & 0 & -1 & 0 \\
0 & 1 & 1 & -1 \\
0 & 0 & 2 k & -k
\end{array}\right) .
$$

As before we see that $k=1$ and the result follows.

We consider a Fano tetrahedron presented in the form given in Proposition 2.1 and assume we are not looking at the case handled in Proposition 2.5. By Proposition 2.4 we have that

$$
\begin{aligned}
\text { either } k-k^{\prime \prime} & =\frac{1+b \lambda_{1}}{\lambda_{3}} \in \boldsymbol{Z} \\
\text { or } \quad k-k^{\prime} & =\frac{1+b \lambda_{2}}{\lambda_{3}} \in \boldsymbol{Z} \\
\text { or } \quad k-k^{\prime}-k^{\prime \prime} & =b \frac{\lambda_{1}+\lambda_{2}}{\lambda_{3}} \in \boldsymbol{Z} .
\end{aligned}
$$

The result of applying this to the barycentric coordinates found in Proposition 1.8 is presented in Table 2. The results of Table 1 and Table 2 complement each other beautifully, allowing the relationships amongst $k, k^{\prime}$ and $k^{\prime \prime}$ shown in Table 3 to be established.

We are now in a position to calculate the vertices of the Fano tetrahedra (up to the action of $G L(3, \boldsymbol{Z})$ ). We will proceed by taking each barycentric coordinate in turn and combining the results of Table 3 and Proposition 2.1. The final results are collected together in Table 4. It is worth comparing this with the results of [Suz02].

Recall that the matrix $\left(x_{1}^{t} \ldots x_{4}^{t}\right)$ is used to represent the tetrahedron with vertices $\left\{x_{1}, \ldots, x_{4}\right\}$. In what follows, references to the vertex $x_{i}$ should be regarded as references to the $i^{\text {th }}$ column of this matrix.

(i) First we consider the case with barycentric coordinate $(1,1,1,1)$. From the results of Table 3 and Proposition 2.1 we have that our Fano tetrahedron has two possible forms, both

TABLE 2. The values depending on $b$.

\begin{tabular}{|c|c|c|c|c|c|}
\hline$\left(\lambda_{1}, \lambda_{2}, \lambda_{3}, \lambda_{4}\right)$ & $a$ & $b$ & $\left(1+b \lambda_{1}\right) / \lambda_{3}$ & $\left(1+b \lambda_{2}\right) / \lambda_{3}$ & $b\left(\lambda_{1}+\lambda_{2}\right) / \lambda_{3}$ \\
\hline \hline$(1,1,1,1)$ & 1 & 0 & \multicolumn{2}{|c|}{1} & 0 \\
\hline$(1,1,1,2)$ & 1 & 0 & \multicolumn{2}{|c|}{1} & 0 \\
\hline$(1,1,2,3)$ & 2 & -1 & \multicolumn{2}{|c|}{0} & -1 \\
\hline$(1,2,3,5)$ & 2 & -1 & 0 & - & -1 \\
\hline$(1,3,4,5)$ & 4 & -3 & - & -2 & -3 \\
\hline$(2,3,5,7)$ & 3 & -2 & - & -1 & -2 \\
\hline$(3,4,5,7)$ & 3 & -2 & -1 & - & - \\
\hline
\end{tabular}


TABLE 3. The relationships among $k, k^{\prime}$ and $k^{\prime \prime}$.

\begin{tabular}{|c|c|c|c|}
\hline$\left(\lambda_{1}, \lambda_{2}, \lambda_{3}, \lambda_{4}\right)$ & $k$ & $k^{\prime}$ & $k^{\prime \prime}$ \\
\hline \hline \multirow{2}{*}{$(1,1,1,1)$} & $k$ & $k-2$ & 2 \\
\cline { 2 - 4 } & $k$ & 3 & $k-1$ \\
\hline \multirow{2}{*}{$(1,1,1,2)$} & $k$ & $k-1$ & 1 \\
\cline { 2 - 4 } & $k$ & $k-1$ & 2 \\
\hline$(1,1,2,3)$ & $k$ & $k$ & 1 \\
\cline { 2 - 4 } & 1 & 1 & 1 \\
\hline$(1,2,3,5)$ & $k$ & 1 & $k$ \\
\hline$(1,3,4,5)$ & $k$ & $k-2$ & 1 \\
\cline { 2 - 4 } & $k$ & $k+2$ & 1 \\
\hline$(2,3,5,7)$ & $k$ & $k+1$ & 1 \\
\hline$(3,4,5,7)$ & $k$ & 2 & $k+1$ \\
\hline
\end{tabular}

of which are equivalent to

$$
\left(\begin{array}{cccc}
1 & 0 & 1 & -2 \\
0 & 1 & -3 & 2 \\
0 & 0 & k & -k
\end{array}\right) .
$$

We observe that $x_{3}$ tells us that $\operatorname{gcd}\{3, k\}=1$ and $x_{4}$ tells us that $\operatorname{gcd}\{2, k\}=1$. Furthermore, taking $k=1$ gives us a tetrahedron equivalent to that found in Proposition 2.2. Suppose that $k \geq 7$. Then $(4 / k) x_{2}+(2 / k) x_{3}+(1 / k) x_{4}=e_{3}$, which contradicts our tetrahedron being Fano. Thus the only remaining possibility is that $k=5$, which by inspection we see does indeed give us a Fano tetrahedron.

(ii-a) Now we consider the case with barycentric coordinate $(1,1,1,2)$. By Table 3 and Proposition 2.1 we see once more that our Fano tetrahedron can take two possible forms. First we consider the form equivalent to

$$
\left(\begin{array}{cccc}
1 & 0 & 1 & -1 \\
0 & 1 & -3 & 1 \\
0 & 0 & 2 k & -k
\end{array}\right)
$$

If we take $k=1$ we obtain a Fano tetrahedron equivalent to that found in Proposition 2.5. Suppose that $k=2$. Then $(1 / 2)(1,-3,4)+(1 / 2)(-1,1,-2)=(0,-1,1)$ is a non-vertex, non-zero lattice point in the interior of the tetrahedron, and hence it is not Fano. The third column tells us that $\operatorname{gcd}\{3, k\}=1$. Finally, the tetrahedron is not Fano if $k \geq 4$ since then $(2 / k) x_{2}+(1 / k) x_{3}+(1 / k) x_{4}=e_{3}$.

(ii-b) Now we consider the second possibility, which is equivalent to

$$
\left(\begin{array}{cccc}
1 & 0 & 3 & -2 \\
0 & 1 & -3 & 1 \\
0 & 0 & 2 k & -k
\end{array}\right)
$$


When $k=1$ we obtain a Fano tetrahedron equivalent to the one previously found. $x_{3}$ and $x_{4}$ tell us that $\operatorname{gcd}\{3, k\}=1$ and $\operatorname{gcd}\{2, k\}=1$, respectively, and if $k \geq 7$ we have the non-vertex, non-zero internal lattice point given by $(3 / k) x_{1}+(1 / k) x_{3}+(3 / k) x_{4}=-e_{3}$. Thus the only remaining possibility is $k=5$, which contains the lattice point $(1 / 5)(1,0,0)+$ $(2 / 5)(3,-3,10)+(1 / 5)(-2,1,-5)=(1,-1,3)$.

(iii) For barycentric coordinate $(1,1,2,3)$ the two possibilities are (up to equivalence)

$$
\left(\begin{array}{cccc}
1 & 0 & 1 & -1 \\
0 & 1 & -2 & 1 \\
0 & 0 & 3 k & -2 k
\end{array}\right) \text { and }\left(\begin{array}{cccc}
1 & 0 & 1 & -1 \\
0 & 1 & 1 & -1 \\
0 & 0 & 3 & -2
\end{array}\right) .
$$

The third column tells us that $k$ must be odd, but if $k \geq 3$ we have the interior lattice point $(1 / k) x_{2}+(1 / k) x_{3}+(1 / k) x_{4}=e_{3}$. Thus the only possibility is that $k=1$, but the resulting tetrahedron is equivalent to that already found.

(iv) When we have barycentric coordinate $(1,2,3,5)$ our tetrahedron equivalent to

$$
\left(\begin{array}{cccc}
1 & 0 & -2 & 1 \\
0 & 1 & 1 & -1 \\
0 & 0 & 5 k & -3 k
\end{array}\right)
$$

The third column tells us that $k$ is odd, and if $k \geq 3$ we have the internal lattice point $(1 / k) x_{1}+$ $(1 / k) x_{3}+(1 / k) x_{4}=2 e_{3}$. By inspection we see that the case where $k=1$ is Fano.

(v-a) For barycentric coordinate $(1,3,4,5)$ we have two possibilities. First we consider the case where our tetrahedron is equivalent to

$$
\left(\begin{array}{cccc}
1 & 0 & 1 & -1 \\
0 & 1 & -22 & 17 \\
0 & 0 & 5 k & -4 k
\end{array}\right)
$$

$x_{3}$ tells us that $k$ is odd. If $k \geq 7$ then it is not Fano, since $(5 / k) x_{2}+(1 / k) x_{3}+(1 / k) x_{4}=e_{3}$. If $k=5$ then $(1 / 5)(1,-22,25)+(1 / 5)(-1,17,-20)=(0,-1,1)$, and if $k=3$ then $k=3$ then $(1 / 3) e_{1}+(1 / 3) e_{2}+(1 / 3)(-1,17,-12)=(0,6,-4)$. By inspection we see that the case where $k=1$ is Fano.

TABLE 4. The vertices of the Fano tetrahedra, up to the action of $G L(3, \boldsymbol{Z})$

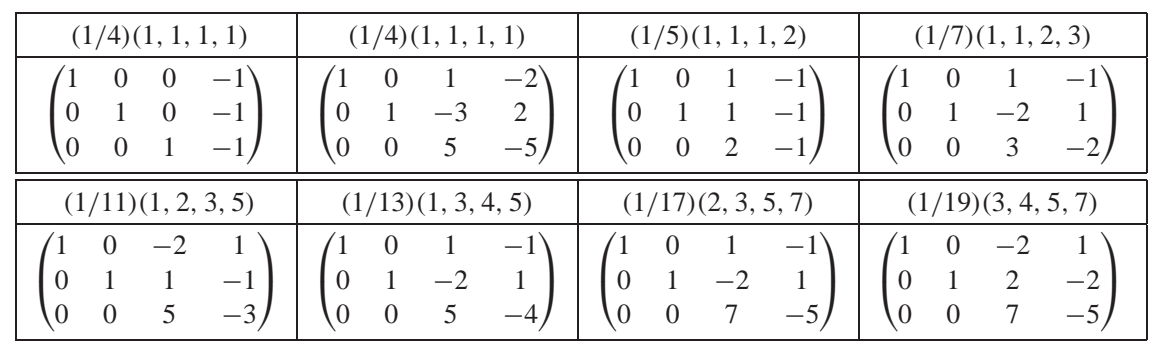


(v-b) The second possibility is the tetrahedron equivalent to

$$
\left(\begin{array}{cccc}
1 & 0 & 1 & -1 \\
0 & 1 & -2 & 1 \\
0 & 0 & 5 k & -4 k
\end{array}\right) .
$$

We require that $k$ is odd, but if $k \geq 3$ we obtain the point $(1 / k) x_{2}+(1 / k) x_{3}+(1 / k) x_{4}=e_{1}$, and when $k=1$ we obtain the tetrahedron found above.

(vi) Continuing in the same vein, for barycentric coordinate $(2,3,5,7)$ we have

$$
\left(\begin{array}{cccc}
1 & 0 & 1 & -1 \\
0 & 1 & -2 & 1 \\
0 & 0 & 7 k & -5 k
\end{array}\right) .
$$

This tells us that $k$ is odd, and if $k \geq 3$ we obtain the internal lattice point $(1 / k) x_{2}+(1 / k) x_{3}+$ $(1 / k) x_{4}=2 e_{3}$. Thus $k=1$ is the only possibility, and we see by inspection that it is indeed Fano.

(vii) Finally consider barycentric coordinate $(3,4,5,7)$.

This gives us

$$
\left(\begin{array}{cccc}
1 & 0 & -2 & 1 \\
0 & 1 & 2 & -2 \\
0 & 0 & 7 k & -5 k
\end{array}\right) .
$$

Once more we see that $k$ must be odd, and that if $k \geq 3$ then it is not Fano since we have $(1 / k) x_{1}+(1 / k) x_{3}+(1 / k) x_{4}=2 e_{3}$. When $k=1$ we do indeed get a Fano tetrahedron.

3. Classifying the minimal polytopes. We extend Definition 1.3 to any polytope $P$.

Definition 3.1. We say a lattice point polytope $P$ in $Z^{3}$ is Fano if $P$ is convex and the only non-vertex lattice point it contains is the origin, which lies strictly in the interior of the polytope.

Given any Fano polytope $P$ with vertices $\left\{x_{1}, \ldots, x_{k}\right\}$ we make the following definition:

Definition 3.2. We say $P$ is minimal if, for all $j \in\{1, \ldots, k\}$, the polytope $P^{\prime}$ given by the vertices $\left\{x_{1}, \ldots, x_{k}\right\} \backslash\left\{x_{j}\right\}$ is not Fano.

Definition 3.3. Let $M=\left\{p_{1}, \ldots, p_{k}\right\}$ be a finite set of points in $N_{Q}$. The convex hull of $M$ is given by $\operatorname{conv} M:=\left\{\sum_{j=1}^{k} v_{j} p_{j} \mid v_{j} \geq 0\right.$ for all $\left.j, \sum_{j=1}^{k} v_{j}=1\right\} \subset N_{Q}$.

Let us consider a minimal Fano polytope $P$. Since $0 \in P$ there exist non-coplanar vertices $x_{1}, \ldots, x_{4}$ of $P$ such that $0 \in \operatorname{conv}\left\{x_{1}, \ldots, x_{4}\right\}=: P^{\prime}$.

Either $P$ is equivalent to one of the tetrahedra in Table 4, or it is not. If it is not, then minimality gives us that it does not contain a Fano tetrahedron; in particular $P^{\prime}$ is not Fano. We assume that this is the case.

Since $P^{\prime}$ is not a Fano tetrahedron it must be that either the origin lies on a face of $P^{\prime}$ or on an edge of $P^{\prime}$. If the origin lies on a face of $P^{\prime}$ then $P$ contains a Fano triangle. Thus there exist three vertices of $P$ which lie in a plane containing the origin, and the origin lies strictly 
in the interior of the triangle defined by these three points. This possibility will be discussed in further detail below.

Assume now that $P$ does not contain a Fano triangle. Then it must be that the origin lies on one of the edges of $P^{\prime}$, say on the edge defined by $x_{1}$ and $x_{2}$. Since the origin lies in the strict interior of $P$ there must exist distinct vertices $y_{1}, \ldots, y_{4}$ of $P$ not equal to $x_{1}$ or $x_{2}$ such that $\operatorname{conv}\left\{x_{1}, x_{2}, y_{1}, y_{2}\right\}$ is a Fano square and $\operatorname{conv}\left\{x_{1}, x_{2}, y_{1}, y_{2}, y_{3}, y_{4}\right\}$ is a Fano octahedron. Minimality gives that $P$ is a Fano octahedron, and these will be classified in Lemma 3.4.

We return now to considering in more detail the case where $P$ contains a Fano triangle, say that defined by $\left\{x_{1}, x_{2}, x_{3}\right\}$. Since the origin lies in the strict interior of $P$ there must exist vertices $y_{1}$ and $y_{2}$ lying on either side of the plane containing our Fano triangle. Minimality then gives us that $P$ is precisely the polygon with vertices $\left\{x_{1}, x_{2}, x_{3}, y_{1}, y_{2}\right\}$.

Now consider the line passing through the origin and $y_{1}$. This line crosses the polytope $P$ at points $y_{1} \in Z^{3}$ and $x$ not necessarily in $\boldsymbol{Z}^{3}$. There are three possible locations for $x$ :

(i) $\quad x$ is equal to $y_{2}$. Then $y_{2}=-y_{1}$. These will be classified in Lemma 3.5.

(ii) $x$ lies on the edge with endpoints $\left\{x_{1}, y_{2}\right\}$. Then $\operatorname{conv}\left\{x_{1}, y_{1}, y_{2}\right\}$ is a Fano triangle. We use the fact that the origin has barycentric coordinate $(1 / 3,1 / 3,1 / 3)$ with respect to $\left\{x_{1}, y_{1}, y_{2}\right\}$. Thus the line passing through $x_{1}$ and the origin bisects the line with endpoints $\left\{y_{1}, y_{2}\right\}$ at a point $x^{\prime}$, say. Now the length of the line joining $\left\{x_{1}, 0\right\}$ is twice the length of the line joining $\left\{x^{\prime}, 0\right\}$. Similarly by considering the Fano triangle $\operatorname{conv}\left\{x_{1}, x_{2}, x_{3}\right\}$, the line passing through $x_{1}$ and the origin bisects the line with endpoints $\left\{x_{2}, x_{3}\right\}$ at a point $x^{\prime \prime}$, say, and we have that the distance from $x_{1}$ to the origin is twice the length of the line joining the origin to $x^{\prime \prime}$. Hence we see that $\left\{x_{2}, x_{3}, y_{1}, y_{2}\right\}$ are coplanar and form a parallelogram. These will be classified in Lemma 3.6.

(iii) $x^{\prime}$ lies strictly in the interior of the triangle $\operatorname{conv}\left\{x_{1}, x_{2}, y_{2}\right\}$. But then $\operatorname{conv}\left\{x_{1}, x_{2}, y_{1}, y_{2}\right\}$ is a Fano tetrahedron, contradicting our assumption.

LEMMA 3.4. The vertices of the minimal Fano octahedra (up to the action of $G L(3, Z)$ ) are given by

$$
\left(\begin{array}{cccccc}
1 & 0 & -1 & 0 & 0 & 0 \\
0 & 1 & 0 & -1 & 0 & 0 \\
0 & 0 & 0 & 0 & 1 & -1
\end{array}\right), \quad\left(\begin{array}{cccccc}
1 & 0 & -1 & 0 & 1 & -1 \\
0 & 1 & 0 & -1 & 1 & -1 \\
0 & 0 & 0 & 0 & 2 & -2
\end{array}\right)
$$

Proof. By making use of Lemma 1.1 and recalling that $P$ does not contain a Fano triangle, we may take the vertices of $P$ to be $\left\{e_{1},-e_{1}, e_{2},-e_{2}, x_{1}, x_{2}\right\}$. We observe that $x_{1}=-x_{2}$, for otherwise we would have that $P$ contains a Fano tetrahedron. So take $x=$ $-x_{2}=x_{1}=(a, b, c)$. First we shall show that, without loss of generality, we may take $a, b, c$ such that

$$
0 \leq a \leq b \leq \frac{c}{2} .
$$

Trivially we may assume that $0 \leq a \leq b$. Suppose that $b>c / 2$. Then $b-c>-c / 2$ and so $c-b<c / 2$. This process corresponds to the action of $G L(3, \boldsymbol{Z})$ transforming 


$$
\left(\begin{array}{cccccc}
1 & 0 & -1 & 0 & a & -a \\
0 & 1 & 0 & -1 & b & -b \\
0 & 0 & 0 & 0 & c & -c
\end{array}\right) \quad \text { to }\left(\begin{array}{cccccc}
1 & 0 & -1 & 0 & a & -a \\
0 & -1 & 0 & 1 & c-b & -(c-b) \\
0 & 0 & 0 & 0 & c & -c
\end{array}\right)
$$

Hence we may assume that the inequality (3.1) holds.

Now consider the point $e_{3}$. Either $x=e_{3}$ or $e_{3}$ lies outside of $P$. The first possibility gives us the first Fano octahedron. The second possibility tells us that $e_{3}$ must lie on the opposite side to the origin of the plane defined by $\left\{-e_{1},-e_{2}, x\right\}$. This plane intersects the $z$-axis at the point $(0,0, c /(a+b+1))$. This gives us that $c \leq a+b$. Combining this with (3.1) gives us that $b \leq a$ and so $b=a$. This in turn gives us that $c \leq 2 b$ and $2 b \leq c$, and so we obtain $2 a=2 b=c$. Thus (up to the action of $G L(3, \boldsymbol{Z})$ ) we have that $a=1, b=1, c=2$, which gives us the second Fano octahedron.

LEMMA 3.5. If $P$ is a minimal Fano polytope with vertices $\left\{x_{1}, x_{2}, x_{3}, y_{1},-y_{1}\right\}$ such that $\left\{x_{1}, x_{2}, x_{3}\right\}$ are the vertices of a Fano triangle, then $P$ is equal (up to the action of $G L(3, Z))$ to one of

$$
\left(\begin{array}{ccccc}
1 & 0 & -1 & 0 & 0 \\
0 & 1 & -1 & 0 & 0 \\
0 & 0 & 0 & 1 & -1
\end{array}\right), \quad\left(\begin{array}{ccccc}
1 & 0 & -1 & 1 & -1 \\
0 & 1 & -1 & 2 & -2 \\
0 & 0 & 0 & 3 & -3
\end{array}\right)
$$

Proof. By making use of Lemma 1.1 we may take the vertices of $P$ to be $\left\{e_{1}, e_{2}\right.$, $\left.-e_{1}-e_{2}, x, y\right\}$. If $y \neq-x$ then $P$ would contain a Fano tetrahedron, which contradicts minimality. Let $x=(a, b, c)$. We claim that, without loss of generality, we may take $a, b, c$ such that $0<a \leq b \leq c$ and

$$
a+b \leq c
$$

Clearly we can take $0<a \leq b$ and $c>0$. Suppose that $a+b>c$. Then we have that $(c-a)+(c-b)<c$. By using the fact that $y=-x$ and applying the transformation

$$
\left(\begin{array}{lll}
1 & 0 & -c \\
0 & 1 & -c \\
0 & 0 & -1
\end{array}\right) \in G L(3, Z)
$$

we see that we may assume that the inequality (3.2) holds.

Now consider the point $e_{3}$. Either $x=e_{3}$ or $e_{3}$ lies outside of $P$. The first case gives us the first Fano polytope in the statement. The second case tells us that we have $e_{3}$ lies on the opposite side to the origin of the plane defined by $\left\{e_{1},-e_{1}-e_{2}, x\right\}$. This plane intersects the $z$-axis at the point $(0,0, c /(2 b-a+1))$, and so

$$
2 b-a \geq c .
$$

Now consider the point $x^{\prime}=e_{2}+e_{3}$. Either $x^{\prime}=x$, which gives a Fano polytope equivalent to the one previously found, or $x^{\prime}$ lies outside of $P$. If this is the case we have that $x^{\prime}$ lies on the opposite side to the origin of the plane defined by $\left\{e_{2},-e_{1}-e_{2}, x\right\}$. This plane intersects the line passing through the origin and $e_{2}+e_{3}$ at the point $(0, k, k)$ where 
$k:=c /(2 a-b+c+1)$. Hence

$$
b \leq 2 a .
$$

Now suppose both $e_{3}$ and $x^{\prime}$ lie outside $P$. Combining the inequalities (3.2) and (3.3) gives us that $2 a \leq b$, and so by (3.4) we obtain that $2 a=b$. Thus (up to the action of $G L(3, Z))$ we have that $a=1, b=2, c=3$. A quick check confirms that this is indeed Fano.

Lemma 3.6. If $P$ is a minimal Fano polytope with vertices $\left\{x_{1}, x_{2}, x_{3}, x_{4}, x_{5}\right\}$ such that $\left\{x_{2}, x_{3}, x_{4}, x_{5}\right\}$ are coplanar and give the vertices of a parallelogram, then $P$ is equal (up to the action of $G L(3, \mathbf{Z})$ ) to

$$
\left(\begin{array}{ccccc}
1 & 0 & -1 & 1 & 0 \\
0 & 1 & -1 & 1 & 0 \\
0 & 0 & 0 & 1 & -1
\end{array}\right) .
$$

Proof. Since $P$ does not contain a Fano tetrahedron it must be that opposite corners of the parallelogram, along with $x_{1}$, give us a Fano triangle. Thus we can (by virtue of Lemma 1.1) write $P$ in the form

$$
\left(\begin{array}{ccccc}
1 & 0 & -1 & a+1 & -a \\
0 & 1 & -1 & b+1 & -b \\
0 & 0 & 0 & c & -c
\end{array}\right)
$$

where $0<a+1 \leq b+1 \leq c$.

Consider the point $-e_{3}$. Either $a=0, b=0, c=1$, which gives the Fano polytope in the statement, or $-e_{3}$ lies outside $P$. Thus $-e_{3}$ lies on the opposite side to the origin of the plane defined by $\left\{e_{1}, e_{2},(-a,-b,-c)\right\}$. This plane intersects the $z$-axis at the point $(0,0, c /(a+b+1))$. Thus we have that $-c>-a-b-1$ and so

$$
c \leq a+b .
$$

Now let $x^{\prime}=e_{1}+e_{2}+e_{3}$. Either $a=0, b=0, c=1$, which gives the Fano polytope in the statement, or $x^{\prime}$ lies outside $P$. Thus $x^{\prime}$ lies on the opposite side to the origin of the plane defined by $\left\{e_{1}, e_{2},(a+1, b+1, c)\right\}$. Thus the plane intersects the line through the origin and $x^{\prime}$ at the point $(k, k, k)$, where $k:=c /(2 c-a-b-1)$. Thus we see that $c<2 c-a-b-1$ and so

$$
c>a+b+1 .
$$

Now suppose both $-e_{3}$ and $x^{\prime}$ lie outside $P$. But then both inequalities (3.5) and (3.6) must be satisfied, which is impossible.

Combining the results of Table 4 and Lemmas 3.4-3.6 we obtain Table 5 .

4. Classifying all Fano polytopes. Given any Fano polytope $P$ with vertices $\left\{x_{1}, \ldots, x_{k}\right\}$ we make the following definition (cf. Definition 3.2): 
TABLE 5. The vertices of the minimal Fano polytopes, up to the action of $G L(3, \boldsymbol{Z})$

\begin{tabular}{|l|ccc|}
\hline Comments & \multicolumn{3}{|c|}{ Vertices } \\
\hline 4 Vertices & $\left(\begin{array}{cccc}1 & 0 & 1 & -2 \\
0 & 1 & -3 & 2 \\
0 & 0 & 5 & -5\end{array}\right)$ \\
\hline Simplicial & $\left(\begin{array}{cccc}1 & 0 & 1 & -1 \\
0 & 1 & -2 & 1 \\
0 & 0 & 7 & -5\end{array}\right)$ \\
\hline 4 Vertices & Simplicial & $\left(\begin{array}{cccc}1 & 0 & -2 & 1 \\
0 & 1 & 2 & -2 \\
0 & 0 & 7 & -5\end{array}\right)$ \\
\hline 4 Vertices & $\left(\begin{array}{cccc}1 & 0 & 0 & -1 \\
0 & 1 & 0 & -1 \\
0 & 0 & 1 & -1\end{array}\right)$ \\
\hline Simplicial & Vertices & $\left(\begin{array}{cccc}1 & 0 & 1 & -1 \\
0 & 1 & -2 & 1 \\
0 & 0 & 5 & -4\end{array}\right)$ \\
\hline 4 Vertices & Simplicial & $\left(\begin{array}{cccc}1 & 0 & -2 & 1 \\
0 & 1 & 1 & -1 \\
0 & 0 & 5 & -3\end{array}\right)$ \\
\hline 4 Vertices & $\left(\begin{array}{cccc}1 & 0 & 1 & -1 \\
0 & 1 & 1 & -1 \\
0 & 0 & 2 & -1\end{array}\right)$ \\
\hline Simplicial Vertices & \\
Simplicial & & & \\
\hline
\end{tabular}

\begin{tabular}{|l|c|}
\hline Comments & Vertices \\
\hline $\begin{array}{l}\text { 4 Vertices } \\
\text { Simplicial }\end{array}$ & $\left(\begin{array}{ccccc|}1 & 0 & 1 & -1 \\
0 & 1 & -2 & 1 \\
0 & 0 & 3 & -2\end{array}\right)$ \\
\hline 5 Vertices & $\left(\begin{array}{ccccc}1 & 0 & 1 & -1 & -1 \\
0 & 1 & 2 & -1 & -2 \\
0 & 0 & 3 & 0 & -3\end{array}\right)$ \\
\hline Simplicial & $\left(\begin{array}{ccccc}1 & 0 & 0 & -1 & 0 \\
0 & 1 & 0 & -1 & 0 \\
0 & 0 & 1 & 0 & -1\end{array}\right)$ \\
\hline S Vertices & $\left(\begin{array}{ccccc}1 & 0 & 1 & -1 & 0 \\
0 & 1 & 1 & -1 & 0 \\
0 & 0 & 1 & 0 & -1\end{array}\right)$ \\
\hline 5 Vertices & $\left(\begin{array}{cccccc}1 & 0 & 1 & -1 & 0 & -1 \\
0 & 1 & 1 & 0 & -1 & -1 \\
0 & 0 & 2 & 0 & 0 & -2\end{array}\right)$ \\
\hline 6 Vertices \\
Simplicial & $\left(\begin{array}{cccccc}1 & 0 & 0 & -1 & 0 & 0 \\
0 & 1 & 0 & 0 & -1 & 0 \\
0 & 0 & 1 & 0 & 0 & -1\end{array}\right)$ \\
\hline 6 Vertices \\
Simplicial
\end{tabular}

Definition 4.1. We say $P$ is maximal if, for all $x_{k+1} \in Z^{3} \backslash\left\{x_{1}, \ldots, x_{k}\right\}$, the polytope $P^{\prime \prime}$ given by the vertices $\left\{x_{1}, \ldots, x_{k}, x_{k+1}\right\}$ is not Fano.

We will also make the following non-standard definition:

Definition 4.2. Let $P=\operatorname{conv}\left\{x_{1}, \ldots, x_{k}\right\}$ and $P^{\prime \prime}$ be Fano polytopes and let $x_{k+1} \in$ $Z^{3}$ be a point such that, up to the action of $G L(3, Z), P^{\prime \prime}=\operatorname{conv}\left\{x_{1}, \ldots, x_{k}, x_{k+1}\right\}$. Then we say that $P$ is the parent of $P^{\prime \prime}$, and that $P^{\prime \prime}$ is the child of $P$.

Clearly a polytope $P$ is minimal if and only if it has no parents, and is maximal if and only if it has no children.

Let $P$ be any Fano polytope. Then the following results are immediate:

(i) Any Fano polytope can be obtained from a (not necessarily unique) minimal Fano polytope by consecutive addition of vertices.

(ii) The number of possible vertices that can be added to $P$ to create a Fano polytope $P^{\prime \prime}$ is finite. For suppose $P$ has vertices $\left\{x_{1}, \ldots, x_{n}\right\}$ and the vertex $x_{n+1}$ is to be added. Then the line through $x_{n+1}$ and the origin, extended in the direction away from $x_{n+1}, \operatorname{crosses} \partial P$ at some point $x^{\prime}$, not necessarily in $Z^{3}$. $x^{\prime}$ corresponds to either a vertex point of $P$, lies on an edge of $P$, or lies on a face. 
TABLE 6. The vertices of the maximal Fano polytopes, up to the action of $G L(3, \boldsymbol{Z})$

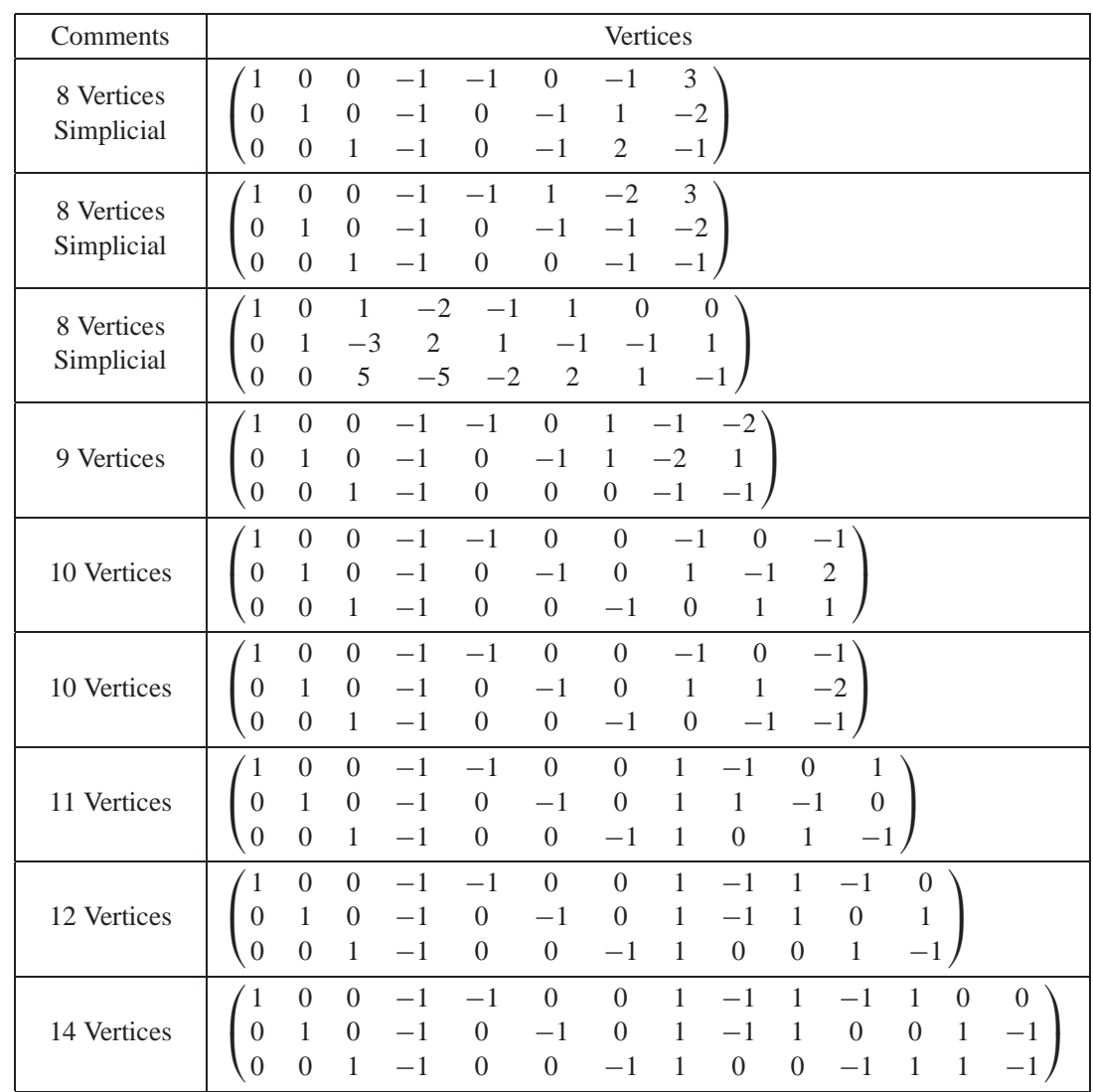

The first possibility gives us that $x_{n+1}=-x_{i}$ for some $i \in\{1, \ldots, n\}$. The second possibility tells us that $\operatorname{conv}\left\{x_{i}, x_{j}, x_{n+1}\right\}$ is an Fano triangle for some distinct $i, j \in\{1, \ldots, n\}$, and hence that $x_{n+1}=-x_{i}-x_{j}$. The final possibility splits naturally into two cases.

The first case corresponds to being able to choose three vertices $x_{i}, x_{j}, x_{k}$ defining the face such that $\operatorname{conv}\left\{x_{i}, x_{j}, x_{k}, x_{n+1}\right\}$ is a Fano tetrahedron (where $i, j, k$ are necessarily distinct, $i, j, k \in\{1, \ldots, n\})$. Hence $\lambda_{\sigma 1} x_{i}+\lambda_{\sigma 2} x_{j}+\lambda_{\sigma 3} x_{k}+\lambda_{\sigma 4} x_{n+1}=0$ for some $\left(\lambda_{1}, \ldots, \lambda_{4}\right)$ in Proposition 1.8 and some $\sigma \in S_{4}$.

The second case corresponds to such a selection being impossible. In this case the face containing $x^{\prime}$ has four vertices which, up to possible renumbering, correspond to the vertices $x_{1}, x_{2}, x_{3}$ and $x_{4}$, and $x^{\prime}$ equals the intersection of the lines joining $x_{1}$ to $x_{3}$ and $x_{2}$ to $x_{4}$. Thus conv $\left\{x_{1}, x_{3}, x_{n+1}\right\}$ is a Fano triangle, and so $x_{n+1}=-x_{1}-x_{3}$ (or, equivalently, equals $\left.-x_{2}-x_{4}\right)$. 
TABLE 7. The number of Fano polytopes in $Z^{3}$.

\begin{tabular}{|l|ccccccccccc|}
\hline Vertices & 4 & 5 & 6 & 7 & 8 & 9 & 10 & 11 & 12 & 13 & 14 \\
\hline \hline Polytopes & 8 & 38 & 95 & 144 & 151 & 107 & 59 & 21 & 8 & 2 & 1 \\
\hline Simplicial & 8 & 35 & 75 & 74 & 35 & 5 & 1 & 0 & 0 & 0 & 0 \\
\hline Minimal & 8 & 3 & 2 & 0 & 0 & 0 & 0 & 0 & 0 & 0 & 0 \\
\hline Maximal & 0 & 0 & 0 & 0 & 3 & 1 & 2 & 1 & 1 & 0 & 1 \\
\hline
\end{tabular}

(iii) If $\left\{x_{1}, \ldots, x_{n}\right\}$ are the vertices of $P$, and the Fano polytope $P^{\prime \prime}$ is created by adding the vertex $x_{n+1}$, then

$$
P^{\prime \prime} \backslash P \subset \bigcup_{i, j} \operatorname{conv}\left\{0, x_{i}, x_{j}, x_{n+1}\right\} .
$$

Using these results and our list of minimal Fano polytopes, it is a relatively straightforward task to write a recursive function to allow a computer to calculate all the Fano polytopes up to the action of $G L(3, Z)$. In particular, (ii) asserts that the calculation will terminate, since the list is finite; a stronger finiteness result to include $\varepsilon$ - $\log$-canonical toric Fano varieties $(0<\varepsilon \leq 1)$ can be found in [BB93, Bor00].

The source code for such a function is available on the Internet at http: //www.maths.bath.ac.uk/ mapamk/code/Polytope_Classify.c.

Using this code a complete classification was obtained in under 20 minutes on an average personal computer. This list, along with a table giving the parents and children of each Fano polytope, is available on the Internet at

http://www.maths.bath.ac.uk/ mapamk/pdf/Fano_List.pdf (or.ps).

The maximal polytopes are reproduced in Table 6 , and a summaries of the results are given in Theorem 0.1 and in Table 7.

\section{REFERENCES}

[BB] A. A. Borisov And L. A. Borisov, Three-dimensional toric Fano varieties with terminal singularities, Text in Russian, Unpublished.

[BB93] A. A. Borisov And L. A. Borisov, Singular toric Fano three-folds, Russian Acad. Sci. Sb. Math. 75 (1993), 277-283.

[Bor00] A. A. Borisov, Convex lattice polytopes and cones with few lattice points inside, from a birational geometry viewpoint, arXiv.math.AG/0001109.

[Cox02] D. A. Cox, Update on toric geometry, Geometry of toric varieties, 1-41, Sémin. Congr. 6, Soc. Math. France, Paris, 2002.

[Dai02] D. I. DAIS, Resolving 3-dimensional toric singularities, Geometry of toric varietics, 155-186, Sémin. Congr. 6, Soc. Math. France, Paris, 2002.

[Dan78] V. I. Danilov, The geometry of toric varieties, Russian Math. Surveys 33 (1978), 97-154.

[Ewa96] G. EwALD, Combinatorial convexity and algebraic geometry, Grad. Texts in Math. 168, Springer, New York, 1996.

[FS03] O. FuJino AND H. SATO, Introduction to the toric Mori theory, arXiv.math.AG/0307180v1. 
[Fu193] W. Fulton, Introduction to toric varieties, Ann. of Math. Stud. 131, Princeton Univ. Press, Princeton, N.J., 1993.

[MMM88] S. MORI, D. R. MORRISON AND I. MORRISON, On four-dimensional terminal quotient singularities, Math. Comp. 51 (1988), 769-786.

[Mor82] S. MoRI, Threefolds whose canonical bundles are not numerically effective, Ann. of Math. (2) 116 (1982), 133-176.

[Mor85] D. R. Morrison, Canonical quotient singularities in dimension three, Proc. Amer. Math. Soc. 93 (1985), 393-396.

[MS84] D. R. MORRISON AND G. STEVEns, Terminal quotient singularities in dimensions three and four, Proc. Amer. Math. Soc. 90 (1984), 15-20.

[Oda78] T. OdA, Torus embeddings and applications, Tata Inst. Fund. Res. Lectures on Math. and Phys. 57, Tata Inst. Fund. Res., Bombay by Springer, Berlin-New York, 1978.

[Rei83] M. REID, Minimal models of canonical 3-folds, Algebraic varieties and analytic varieties, (Tokyo, 1981), 131-180, Adv. Stud. Pure Math. 1, North-Holland, Amsterdam, 1983.

[Rei85] M. REID, Young person's guide to canonical singularities, Algebraic Geometry, Bowdoin, 1985 (Brunswick, Maine, 1985), 345-414, Proc. Sympos. Pure Math. 46, pt. 1, Amer. Math. Soc., Providence, R.I., 1987.

[Sca85] H. E. SCARF, Integral polyhedra in three space, Math. Oper. Res. 10 (1985), 403-438.

[Suz02] K. SuzUKI, On Fano indicies of $\boldsymbol{Q}$-fano 3-folds, arXiv.math.AG/0210309v1.

[Wiś02] J. A. WiśnIEWski, Toric Mori theory and fano manifolds, Geometry of toric varieties, 249-272, Sémin. Congr. 6, Soc. Math. France, Paris, 2002.

DPARTMENT OF MATHEMATICAL SCIENCES

UNIVERSITY OF BATH

BATH, BA2 7AY

UNITED KINGDOM

E-mail address: A.M.Kasprzyk@maths.bath.ac.uk 